\title{
Design and Implementation of Wireless Healthcare Monitoring System
}

\author{
Kalpesh Patel \\ M.E (Embedded System) \\ Electronics \& Communication Dept \\ G.H.Patel College of Engineering \& Technology \\ Anand, India
}

\author{
Rahul Kher, Ph.D \\ Associate Professor, \\ Electronics \& Communication Dept \\ G.H.Patel college of engineering \& Technology \\ Anand, India
}

\begin{abstract}
Healthcare monitoring is now becoming part of everyday life. Today's healthcare industry aims to provide better healthcare to people anywhere, anytime in the world in economical and patient friendly manner. In this paper the physiological parameters like heart rate, body temperature, $\mathrm{SpO}_{2}$ and ECG signal are obtained and displayed using PIC18F45K22 Microcontroller. Body temperature, $\mathrm{SpO}_{2}$ and heart rate are displayed on LCD display and ECG signal is monitored on PC through Labview. If the abnormalities in the recorded parameters are detected, then an SMS alert will be sent to the physician. The system comprises of low cost ECG, $\mathrm{SpO}_{2}$ and temperature sensors along with the GSM modem.
\end{abstract}

\section{Keywords}

ECG sensor, Temperature sensor, PIC microcontroller, GSM module

\section{INTRODUCTION}

The embedded system (electronics technology) has entered almost in all aspects of today's life and medical area is not exception for that. The need for well-equipped hospitals and healthcare centers is increasing day by day as the people are becoming more aware about their health problems. In some hospitals special units are used for biomedical field, as for example such as intensive care unit or coronary care unit. All of these units are designed, so that the advantage of the low nursepatient ratio, concentration of the equipment and their resources needed, to take care of critically ill or seriously injured units. Today medical field faces two basic problems when it comes to patient monitoring. First one, the presence of healthcare provider beside the patient and second, the patient is restricted to bed and wired to large machines.[2] With the technological advancement, it has become possible to design low-cost, home based healthcare monitoring system in which the system records and displays the signals from human body and transmits to any other location. This paper discusses the acquisition of physiological parameters such as ECG \& heart rate, body temperature, $\mathrm{SpO}_{2}$ (pulse oximetory) and displaying them on LCD display/PC.

\section{DETAILED DESCRIPTION OF SYSTEM}

This section includes:

- Block diagram of the system

- Flowchart

- Algorithm

\subsection{Block diagram}

The blocks in the diagram represent the major components of the system and interconnection between them.

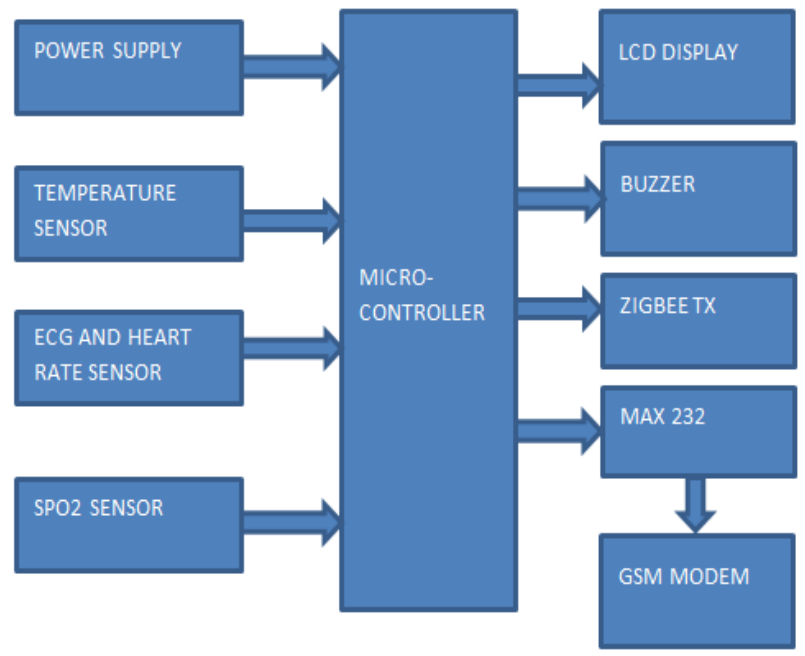

Fig.1 Block diagram of system

- Sensors: Sensors like temperature sensor, $\mathrm{SpO}_{2}$ sensor, ECG sensor are placed on the patient's body.

- PIC microcontroller: It receives the outputs of the sensors and analyzes them. Then send them through zigbee transmitter to physician PC. It also sends an SMS alert to physician mobile through GSM. [5]

- $\quad$ ZIGBEE module: zigbee module is used for wireless data transmission between microcontroller and PC.[6]

- GSM module: It sends a message or call to doctor's mobile.

\subsection{Flowchart}

Flowchart describes the flow of control in the system.

\subsection{Algorithm}

This describes the flow of system operation

Various sensors are placed on human's body to collect the real time data about his health.

This collected data are then compared with the threshold data to check the abnormality of the patient.

Database of the patients are continuously updated in doctor's PC. 
Whenever abnormal condition is detected, an alarm is also sent to the doctor's mobile from of a SMS alert, through GSM modem [7].

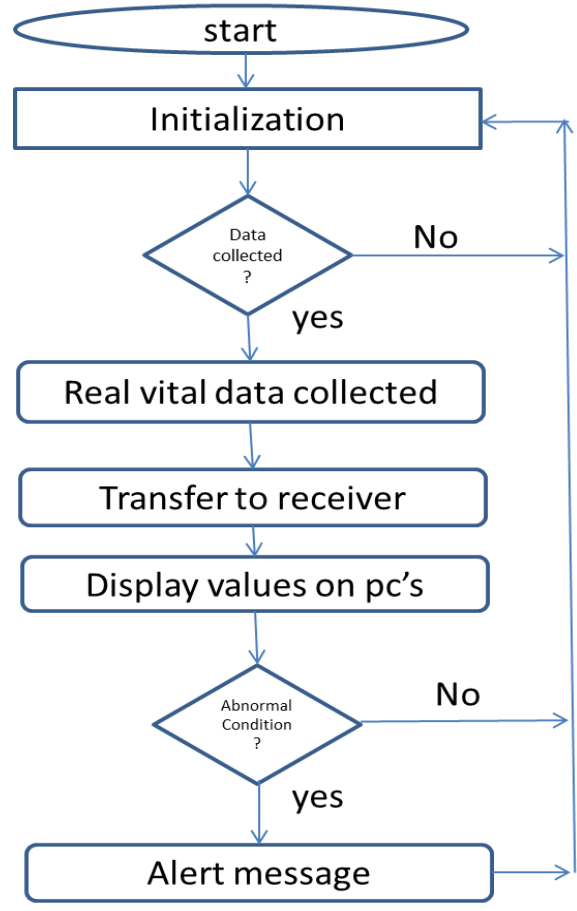

\section{Fig.2 Flowchart of system}

Normal range for various parameters:

The body temperature lies in the range 36.5 degrees $\mathrm{C}$ to 37.2 degrees $\mathrm{C}$ for a healthy person. The heart rate of a normal adult ranges from 60 to 100 beats per minute. Form ECG waveform many cardiac abnormalities can be predicted through its various intervals, shapes and morphologies. ECG is the most common test for heart conditions. It is a simple painless test that takes few minutes. An ECG machine records heart's rhythm onto paper through sticky electrodes which are placed on human chest, arms and legs.[3] The recording will show if the heart muscle is damaged or short of oxygen.

\section{METHODOLOGY}

Designing part consists basically two sections.

\subsection{Hardware}

PIC microcontroller: The blocks in the diagram represent the major components of the system and interconnection between them

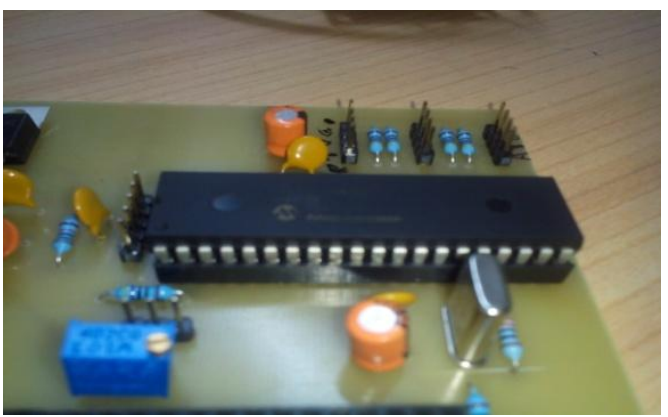

Fig.3 PIC microcontroller PIC18F45K22
Temperature sensor: LM335 sensor is used for temperature measurement, whose output voltage is linearly proportional to the Celsius (centigrade) temperature.

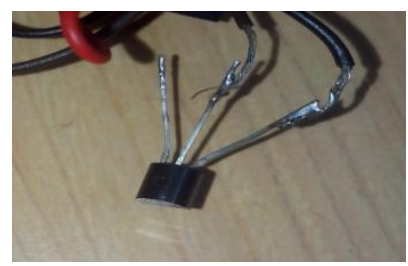

Fig.4 Temperature sensor

ECG sensor: For ECG measurement electrodes are placed on human body as shown in fig. 5 to obtain small electrical voltage produced through contracting muscles because of each heart rate. The ECG signal captured by the electrodes is in the voltage range of $1 \mathrm{mv}$ to $5 \mathrm{mv}$. Because of this weak voltage level, the signal is fed in to an instrumentation amplifier to amplify the acquired signal. The amplified signal is then fed in to the PIC18F45K22 having inbuilt $\mathrm{A} / \mathrm{D}$ converter. Digital output of the ADC can be seen on the labView software via serial port transmission.

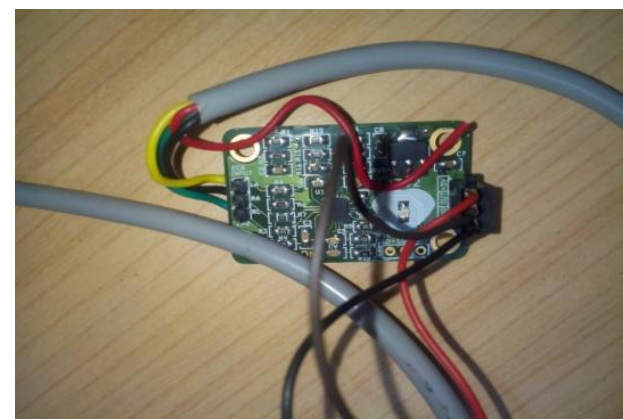

Fig.5 ECG sensor

\subsection{Software}

Coding of PIC microcontroller is done in MPLAB IDE software. MPLAB IDE is a software program that is used to develop application for microcontrollers and digital signal controllers.

Following are the steps for creating a new project file in MPLAB:

- $\quad$ Project > Project Wizard then Click Next

- $\quad$ Select Device from Drop down Menu then Click Next

- $\quad$ Active Tool suit > Microchip C18 Tool suit

- Once you select C18 Tool suit, check if it shows Red Alert.

- Then Tick and select "Store tool locations in project"

- Finally it will show summary page. Click Finish.

- Now you will see a window with source files and header files etc.

- Now click on Project > Add New File to Project.

- $\quad$ Save file with .c extension

- Finally write code for system in this .c extension file. 


\subsection{System Design Parameters}

The AD8232 is an integrated signal conditioning block. AD8232 used in ECG and other biopotential measurement applications. It is designed to extract, amplify, and filter signal in the presence of noisy conditions, like as remote electrode placement. By using AD8232, one can implement a two-pole high-pass filter for removing motion artifacts and the electrode half-cell potential. This filter is strongly joined with the instrumentation architecture of the amplifier to allow both large gain and highpass filtering in a single stage, thereby saving space and cost. An operational amplifier turns on the AD8232 to create a three-pole low-pass filter to remove additional noise. Thus the user can select the cutoff frequency of all filters to suit different types of applications.

In instrumentation amplifier CMRR value (80-86 dB), PSRR (76-90 dB), gain 100, bandwidth $2 \mathrm{kHz}$, RFI filter cutoff (each input) $1 \mathrm{MHz}$ and in operational amplifier CMRR and PSRR both $100 \mathrm{~dB}$. large signal voltage gain $110 \mathrm{~dB}$. In AD8232 twopole high pass filter at $7 \mathrm{~Hz}$ and two-pole low pass filter at $24 \mathrm{~Hz}$ have been used. The two pole low pass filter follows the two pole high pass filters to eliminate any other artifacts and line noise.

LabVIEW is a short form of laboratory Virtual instrument Engineering Workbench. LabVIEW support interfacing to devices, such as cameras, and other devices. LabVIEW is a platform and development environment for visual programming language from NI (National Instruments) and uses graphical programming language. In the system implemented in this paper, LabVIEW is interfaced with microcontroller through serial port a virtual port for communication between microcontroller and PC. Real time ECG signal is continuously monitored using LabVIEW software.

\section{RESULTS}

Figure 6 shows the body temperature and heart rate (37 degree celcius and 60 beats/minute, respectively) of the subject displayed on LCD. Figure 7 shows the captured ECG signal of the subject with lead I configuration on DSO. The same is being monitored on PC as well using Labview.

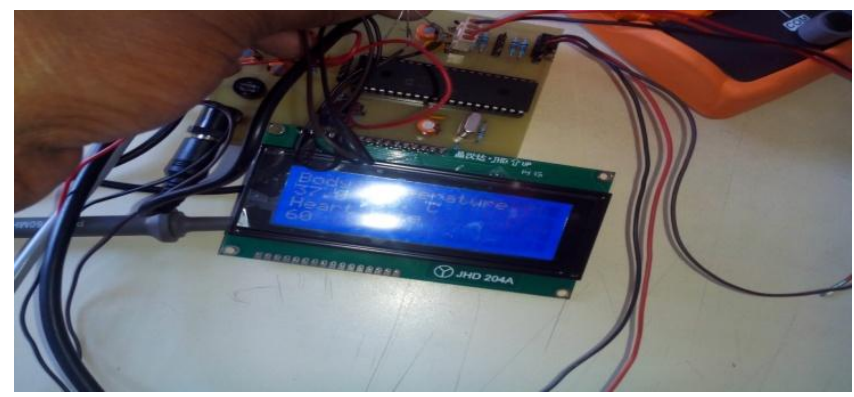

Fig.6 Tempearture and heart rate diplayed on LCD

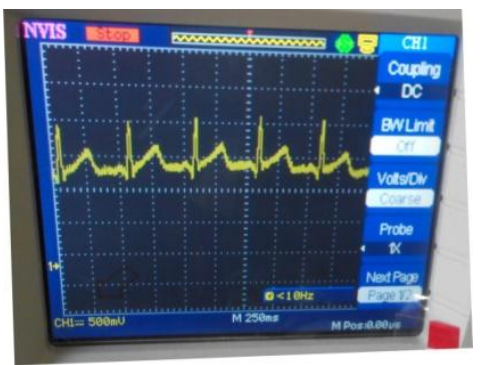

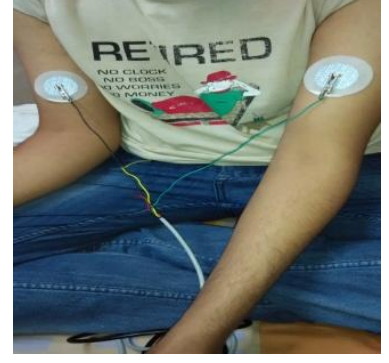

Fig.7 ECG waveform display on oscilloscope

\section{CONCLUSION AND FUTURE WORK}

In this paper, the physiological parameters such as body temperature, ECG and heart rate of the subject are monitored and displayed. Whenever the parameters such as body temperature or heart rate goes below or above from the normal range then an alert message or call to the physician will be generate by using GSM module. In future, the pulse oximetory (SpO2) sensor which measures the percentage of oxygen in blood of the subject will be interfaced with the microcontroller. A web page will be created, where these physiological data of the subject(s) will be continuously transferred by incorporating the zigbee based wireless communication. Thus, a physician at distant location can access the physiological data of the subjects.

\section{REFERENCES}

[1] Richard R. Fletcher, Ming-Zher Poh, "Wearable Sensors: Opportunities and Challenges for Low-Cost Health care", $32^{\text {nd }}$ Annual International Conference of the IEEE EMBS Buenos Aires, Argentina, August 31-Sep.4, 2010.

[2] Bhargavi Sundara, Dr. Kalyana Chakravarthi Sarvepalli, Sri Harsha Davuluri, "GSM Based Patient Monitoring System in ICU”, IJRET: International Journal of Research in Engineering and Technology, Volume: 02 Issue: 07, Jul2013.

[3] N. M. Z. Hashim, M. S. Sizali "Wireless Patient Monitoring System", International Journal of Science and Research (IJSR), India, Volume 2 Issue 8, August 2013.

[4] Aditi Agrawal, Pavan kumar Reddy, Santha MeenaI, "An Embedded system for determining arrhythmia", International Conference on Computer Communication and Networking Technologies ICCCNT'12, 26 - 28 July 2012, Coimbatore, India.

[5] Syed Faraz Jawed, Muhammad Owais Khan, Osama Mazhar, "An Innovative Approach towards E-health in Development of Tele Auscultation System for Heart using GSM Mobile Communication Technology" 2013 IEEE $19^{\text {th }}$ International Symposium for Design and Technology in Electronic Packaging (SIITME), 24-27 Oct 2013, Galați, Romania.

[6] Sowmyasudhan S, Manjunath S, "A Wireless Based Realtime Patient Monitoring System", International Journal of Scientific \& Engineering Research, Volume 2, Issue 11, November-2011.

[7] Eriola J. Shanko, Michalis G. Papoutsidakis, "Real Time Health Monitoring and Wireless Transmission: A microcontroller Application to Improve Human Medical Needs", The 4th IEEE International Conference on EHealth and Bioengineering - EHB 2013, Romania, November 21-23, 2013. 
[8] Divyesh Dixit, Ankit Kalbande, K.M Bhurchandi, "RFID Based Health Assistance \& Monitoring System through a Handmounted Embedded Device", $4^{\text {th }}$ International Conference on Computer Communication and Networking Technologies ICCCNT 2013 July 4-6, 2013, Tiruchengode, India
[9] Pengxiang Jin, Chao Hu, Weixing Lin, "Interface design for multi-parameter health monitoring instrument based on ARM9 embedded system" ,Proceeding of the IEEE International Conference on Information and Automation Hailar ,China , July 2014

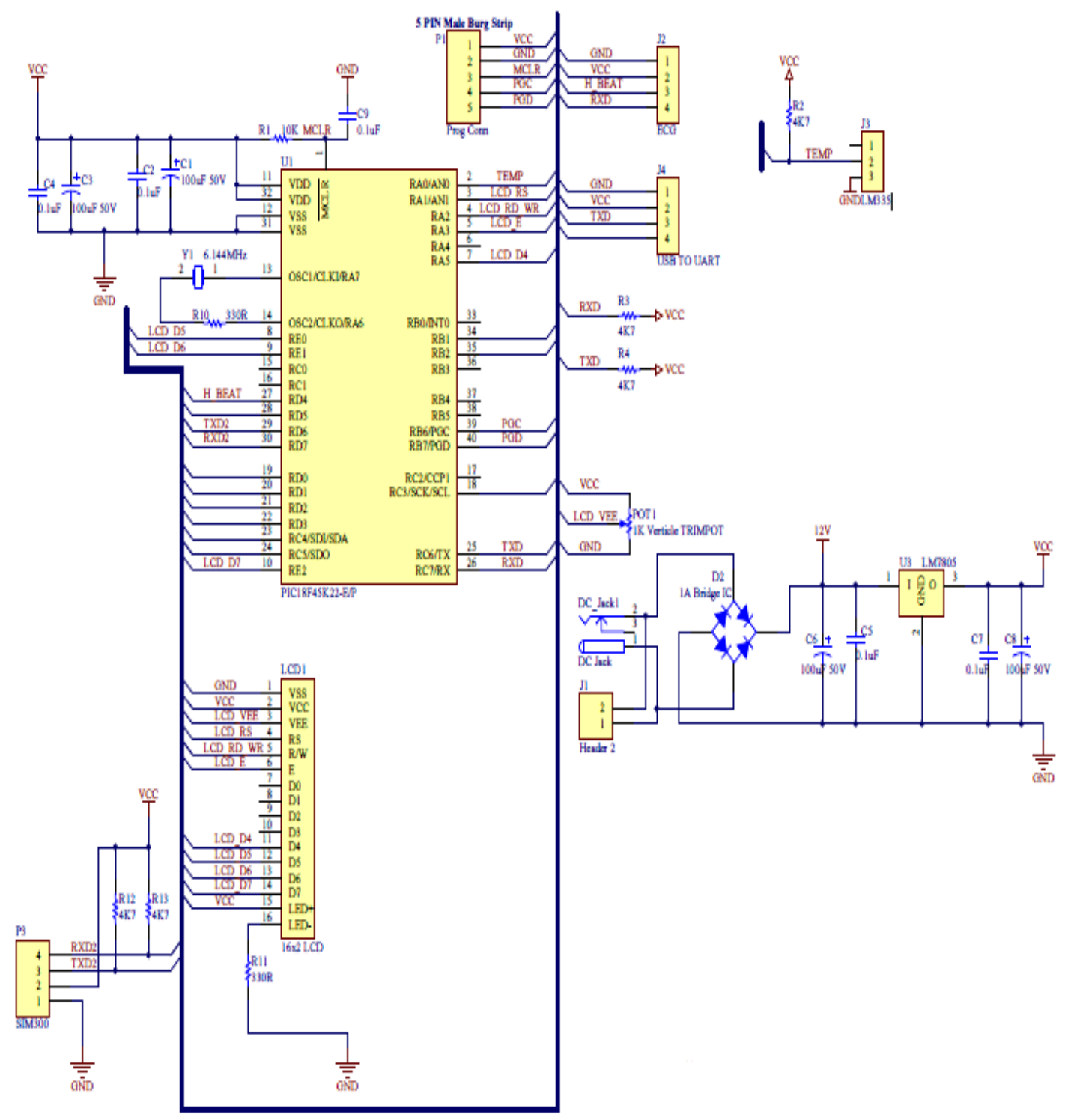

Fig.8 Schematic diagram of system 